\title{
Polyphenol metabolomics of twenty Italian red grape varieties
}

\author{
Luigi Bavaresco ${ }^{1, \text { a }}$, Mirko De Rosso ${ }^{2}$, Massimo Gardiman $^{2}$, Giacomo Morreale $^{2}$, and Riccardo Flamini ${ }^{2}$ \\ ${ }^{1}$ Dept. of Sustainable Crop Production, Università Cattolica S.C., via Emilia Parmense 84, 29122 Piacenza, Italy \\ ${ }^{2}$ Consiglio per la ricerca in agricoltura e l'analisi dell'economia agraria, Centro di Ricerca per la viticoltura (CREA-VIT), \\ viale XXVIII Aprile 26, 31015 Conegliano, Italy
}

\begin{abstract}
Suspect screening analysis"method to study grape metabolomics, was performed. This method is a middle-way "targeted" and "untargeted"approach aiming at identifying the largest number of metabolites in grape samples. A new database of putative grape and wine metabolites (GrapeMetabolomics), which currently contains around 1,100 compounds, was constructed by CREA at Conegliano. By performing high-resolution mass spectrometry analysis of the grape extract in both positive and negative ionization mode, averaging 320450 putative compounds are identified. Most of them are grape polyphenols, such as anthocyanins, flavonols and stilbene derivatives. By performing PCA and Cluster Analysis the composition in anthocyanins and flavonols of 20 Italian red grape varieties, was studied.
\end{abstract}

\section{Introduction}

Anthocyanins are polyphenolic compounds found in the grape skin that are responsible for the red color of the grapes and wine. These secondary metabolites play a key role both in terms of sensory profile and in terms of the health value, with antioxidant, antimicrobial, anticancer effects and protection of the cardiovascular system (De Pascual-Teresa et al., 2008).

The anthocyanins may also be used as natural dyes in food industry and as useful compounds for the pharmaceutical and nutraceutical industries.

$V$. vinifera flavonols are an interesting class of polyphenols both studied for their copigmentation with anthocyanins in red wines (Boulton, 2001) and for food and health implications (Manach al., 2004). Anthocyanins and flavonols are also widely studied for variety characterization (Mattivi et al., 2006).

In this work, a suspect screening analysis method developed for the grape metabolomics (Flamini et al., 2013) was used to study anthocyanins and flavonols of twenty Italian native red grape varieties. The relationship between variety and polyphenolic composition was studied by multivariate statistical analysis (PCA and Cluster Analysis).

\section{Materials and methods}

Red grape varieties Aglianico, Barbera, Cannonau, Cesanese d'Affile, Corvina, Enantio, Grignolino, Lambrusco Grasparossa, Montepulciano, Nebbiolo, Negroamaro, Nero d'Avola, Primitivo, Raboso Piave, Refosco dal Peduncolo Rosso, Rossese, Sagrantino, Sangiovese, Terrano, and Uva di Troia, were studied.

\footnotetext{
${ }^{a}$ Corresponding author: luigi.bavaresco@unicatt.it
}

For each variety about 100 grape berries were collected at physiological maturity from the CREAVIT grapevine germplasm collection (Susegana, TV, harvest 2013). Twenty berries were weighed. Following seed removal, the sample was homogenized using liquid nitrogen and the resulting powder immediately extracted with methanol ratio $2: 1(\mathrm{v} / \mathrm{w})$ under stirring for $20 \mathrm{~min}$. After the addition of $200 \mu \mathrm{L}$ of $4^{\prime}, 5,7$-trihydroxy flavanone $(500 \mathrm{mg} / \mathrm{L})$ as internal standard, the sample was centrifuged at $4000 \mathrm{~g} / \mathrm{min}\left(10^{\circ} \mathrm{C}\right)$ for $20 \mathrm{~min}$. The supernatant was filtered on $0.22 \mu \mathrm{m}$ filter and subjected to LC/MS analysis. Each sample was replicated twice. The analyses were performed in both negative and positive ionization mode by using a ultra-performance Agilent 1290 Infinity liquid chromatography system coupled to a high resolution time of flight mass spectrometer AgilentQ TOF 6540 (40,000 nominal resolution). The gradient chromatographic separation was performed on a reversed-phase column Zorbax (RRHD SB-C18 $150 \times$ $3 \mathrm{~mm}, 1.8 \mu \mathrm{m}$ ) and mobile phase composed of A) aqueous $0.1 \% \mathrm{v} / \mathrm{v}$ formic acid, B) acetonitrile containing $0.1 \% \mathrm{v} / \mathrm{v}$ formic acid, at a flow rate $0.4 \mathrm{~mL} / \mathrm{min}$ Sample injection $10 \mu \mathrm{L}$. Settings of QTOF mass spectrometer: nitrogen sheath gas flow $10 \mathrm{~L} / \mathrm{min}$ at $400{ }^{\circ} \mathrm{C}$; nitrogen drying gas flow $8 \mathrm{~L} / \mathrm{min}$ at $350{ }^{\circ} \mathrm{C}$; nebulizer pressure $60 \mathrm{psi}$; cone voltage $1 \mathrm{kV}$ (positive), $0 \mathrm{kV}$ (negative); capillary voltage $3.5 \mathrm{kV}$. The signals were recorded in the $\mathrm{m} / \mathrm{z}$ range 100 1700. Statistical analysis was performed using the software PAST 3.13 (Hammer et al., 2001).

\section{Results and discussion}

The utilization of database GrapeMetabolomics allowed the identification of 16 anthocyanins and 15 flavonols in each V. vinifera sample. This database of metabolites

(C) The Authors, published by EDP Sciences. This is an Open Access article distributed under the terms of the Creative Commons Attribution License 4.0 (http://creativecommons.org/licenses/by/4.0/). 
Table 1. Anthocyanins identified in $V$. vinifera grape extracts.

\begin{tabular}{|l|l|l|l|}
\hline \multicolumn{1}{|c|}{ Anthocyanin } & $\begin{array}{l}\text { RT } \\
\text { (min) }\end{array}$ & Formula & $\begin{array}{l}\mathbf{M}^{+} \\
(\mathbf{m} / z)\end{array}$ \\
\hline $\begin{array}{l}\text { Delphinidin-3- } O \text { - } \\
\text { glucoside }\end{array}$ & 12.03 & $\mathrm{C}_{21} \mathrm{H}_{21} \mathrm{O}_{12}$ & 465.1028 \\
\hline $\begin{array}{l}\text { Cyanidin-3- } O \text { - } \\
\text { monoglucoside }\end{array}$ & 12.57 & $\mathrm{C}_{21} \mathrm{H}_{21} \mathrm{O}_{11}$ & 449.1078 \\
\hline $\begin{array}{l}\text { Petunidin-3- } O \text { - } \\
\text { monoglucoside }\end{array}$ & 12.74 & $\mathrm{C}_{22} \mathrm{H}_{23} \mathrm{O}_{12}$ & 479.1184 \\
\hline $\begin{array}{l}\text { Peonidin-3- } O- \\
\text { monoglucoside }\end{array}$ & 13.12 & $\mathrm{C}_{22} \mathrm{H}_{23} \mathrm{O}_{11}$ & 463.1240 \\
\hline $\begin{array}{l}\text { Malvidin-3- } O- \\
\text { monoglucoside }\end{array}$ & 13.27 & $\mathrm{C}_{23} \mathrm{H}_{25} \mathrm{O}_{12}$ & 493.1341 \\
\hline $\begin{array}{l}\text { Delphinidin-3- } O-(6-O- \\
\text { acetyl)monoglucoside }\end{array}$ & 13.59 & $\mathrm{C}_{23} \mathrm{H}_{23} \mathrm{O}_{13}$ & 507.1133 \\
\hline $\begin{array}{l}\text { Cyanidin-3- } O-(6-O- \\
\text { acetyl)monoglucoside }\end{array}$ & 14.06 & $\mathrm{C}_{23} \mathrm{H}_{23} \mathrm{O}_{12}$ & 491.1184 \\
\hline $\begin{array}{l}\text { Petunidin-3- } O \text { - }(6-O- \\
\text { acetyl)monoglucoside }\end{array}$ & 14.14 & $\mathrm{C}_{24} \mathrm{H}_{25} \mathrm{O}_{13}$ & 521.1290 \\
\hline $\begin{array}{l}\text { Malvidin-3- } O-(6-O- \\
\text { acetyl)monoglucoside }\end{array}$ & 14.64 & $\mathrm{C}_{25} \mathrm{H}_{27} \mathrm{O}_{13}$ & 535.1446 \\
\hline $\begin{array}{l}\text { Peonidin-3- } O-(6-O- \\
\text { acetyl)monoglucoside }\end{array}$ & 14.65 & $\mathrm{C}_{24} \mathrm{H}_{25} \mathrm{O}_{12}$ & 505.1341 \\
\hline $\begin{array}{l}\text { Delphinidin-3-(6- } O-p- \\
\text { coumaroyl)monoglucoside }\end{array}$ & 14.82 & $\mathrm{C}_{30} \mathrm{H}_{27} \mathrm{O}_{14}$ & 611.1395 \\
\hline $\begin{array}{l}\text { Malvidin-3-(6- } O- \\
\text { caffeoyl)monoglucoside }\end{array}$ & 15.14 & $\mathrm{C}_{31} \mathrm{H}_{31} \mathrm{O}_{15}$ & 655.1663 \\
\hline $\begin{array}{l}\text { Cyanidin-3-(6- } O-p- \\
\text { coumaroyl)monoglucoside }\end{array}$ & 15.24 & $\mathrm{C}_{30} \mathrm{H}_{27} \mathrm{O}_{13}$ & 595.1446 \\
\hline $\begin{array}{l}\text { Petunidin-3-(6- } O-p- \\
\text { coumaroyl)monoglucoside }\end{array}$ & 15.30 & $\mathrm{C}_{31} \mathrm{H}_{29} \mathrm{O}_{14}$ & 625.1552 \\
\hline $\begin{array}{l}\text { Malvidin-3-(6- } O-p- \\
\text { coumaroyl)monoglucoside }\end{array}$ & 15.77 & $\mathrm{C}_{32} \mathrm{H}_{31} \mathrm{O}_{14}$ & 639.1708 \\
\hline $\begin{array}{l}\text { Peonidin-3-(6- } O-p- \\
\text { coumaroyl)monoglucoside }\end{array}$ & 15.78 & $\mathrm{C}_{31} \mathrm{H}_{29} \mathrm{O}_{13}$ & 609.1603 \\
\hline
\end{tabular}

contains approximately 1,100 compounds and was expressly constructed to study grape metabolomics (Flamini et al., 2015). Anthocyanins identified in positive ion mode were delphinidin, cyanidin, petunidin, peonidin, and malvidin in monoglucoside, acetylmonoglucoside and p-coumaroyl-monoglucoside forms. Malvidin caffeoyl-monoglucoside was also identified. Compounds are reported in Table 1 with their $\mathrm{M}+\mathrm{m} / \mathrm{z}$ signal.

Flavonols were detected in negative ion mode, the compounds identified are reported in Table 2 with their $[\mathrm{M}-\mathrm{H}]^{-} m / z$ signal. Among them also B-ring trisubstituted glycosides were identified (i.e. myricetin, larycitrin and syringetin), which are typical of red grape varieties.

Due to the lack of standards available, semiquantitative analysis was performed by normalization of the signal area with that of the internal standard. This method allowed the comparison among the different varieties. Multivariate analysis (PCA and cluster analysis) to study the effect of variety on these secondary metabolites, was performed, and results are shown in the Fig. 1 and Fig. 2.

As may be seen from the figures, was possible classify the samples into five groups on the basis of their polyphenolic profile: A) Rossese, B) Aglianico, Cannonau,
Table 2. Flavonols identified in $V$. vinifera grape extracts.

\begin{tabular}{|l|l|l|l|}
\hline \multicolumn{1}{|c|}{ Flavonols } & $\begin{array}{l}\text { RT } \\
(\text { min })\end{array}$ & Formula & $\begin{array}{l}{[\mathbf{M}-\mathrm{H}]^{-}} \\
(\mathbf{m} / z)\end{array}$ \\
\hline $\begin{array}{l}\text { Myricetin-3- } O \text { - } \\
\text { glucuronide }\end{array}$ & 14.26 & $\mathrm{C}_{21} \mathrm{H}_{18} \mathrm{O}_{14}$ & 493.0624 \\
\hline Myricetin-3- $O$-glucoside & 14.31 & $\mathrm{C}_{21} \mathrm{H}_{20} \mathrm{O}_{13}$ & 479.0831 \\
\hline $\begin{array}{l}\text { Dihydroquercetin-3- } O \text { - } \\
\text { hexoside }\end{array}$ & 14.40 & $\mathrm{C}_{21} \mathrm{H}_{22} \mathrm{O}_{12}$ & 465.1038 \\
\hline Rutin & 14.68 & $\mathrm{C}_{27} \mathrm{H}_{30} \mathrm{O}_{16}$ & 609.1461 \\
\hline Quercetin-3- $O$-galactoside & 15.00 & $\mathrm{C}_{21} \mathrm{H}_{20} \mathrm{O}_{12}$ & 463.0882 \\
\hline $\begin{array}{l}\text { Quercetin-3- } O \text { - } \\
\text { glucuronide }\end{array}$ & 15.02 & $\mathrm{C}_{21} \mathrm{H}_{18} \mathrm{O}_{13}$ & 477.0675 \\
\hline Larycitrin-3- $O$-hexoside & 15.06 & $\mathrm{C}_{22} \mathrm{H}_{22} \mathrm{O}_{13}$ & 493.0988 \\
\hline Quercetin-3- $O$-glucoside & 15.06 & $\mathrm{C}_{21} \mathrm{H}_{20} \mathrm{O}_{12}$ & 463.0882 \\
\hline $\begin{array}{l}\text { Dihydroquercetin-3- } O- \\
\text { rhamnoside }\end{array}$ & 15.34 & $\mathrm{C}_{21} \mathrm{H}_{2} 2 \mathrm{O}_{11}$ & 449.1089 \\
\hline $\begin{array}{l}\text { Kaempferol-3- } O- \\
\text { galactoside }\end{array}$ & 15.47 & $\mathrm{C}_{21} \mathrm{H}_{20} \mathrm{O}_{11}$ & 447.0933 \\
\hline Kaempferol-3-O-glucoside & 15.65 & $\mathrm{C}_{21} \mathrm{H}_{20} \mathrm{O}_{11}$ & 447.0933 \\
\hline $\begin{array}{l}\text { Kaempferol-3- } O- \\
\text { glucuronide }\end{array}$ & 15.66 & $\mathrm{C}_{21} \mathrm{H}_{18} \mathrm{O}_{12}$ & 461.0725 \\
\hline Syringetin-3- $O$-glucoside & 15.73 & $\mathrm{C}_{23} \mathrm{H}_{24} \mathrm{O}_{13}$ & 507.1144 \\
\hline $\begin{array}{l}\text { Isorhamnetin-3- } O- \\
\text { hexoside }\end{array}$ & 15.78 & $\mathrm{C}_{22} \mathrm{H}_{22} \mathrm{O}_{12}$ & 477.1038 \\
\hline $\begin{array}{l}\text { Dihydrokaempferol-3- } O- \\
\text { ramnoside }\end{array}$ & 16.08 & $\mathrm{C}_{21} \mathrm{H}_{22} \mathrm{O}_{10}$ & 433.1140 \\
\hline
\end{tabular}

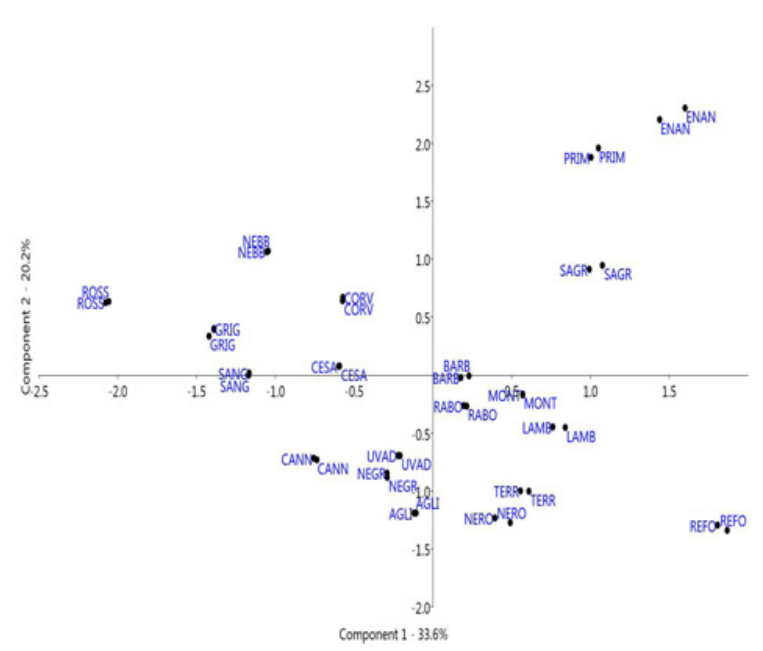

Figure 1. PCA analysis performed by using anthocyanin and flavonol signals measured in the extracts of the 20 red grape varieties studied.

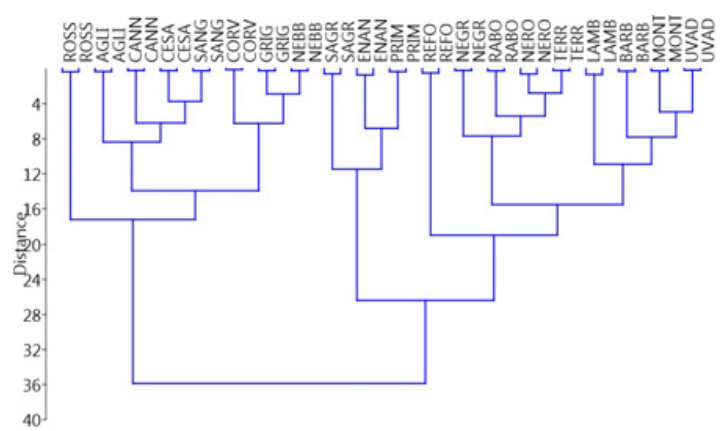

Figure 2. Hierarchical clustering analysis (Ward's method, Euclidean similarity index) performed by using anthocyanin and flavonol signals measured in the extracts of the 20 red grape varieties studied. 
Cesanese d'Affile, Sangiovese, Corvina, Grignolino, Nebbiolo, C) Sagrantino, Enantio, Primitivo, D) Refosco dal peduncolo rosso, E) Negroamaro, Raboso Piave, Nero d'Avola, Terrano, Lambrusco Grasparossa, Barbera, Montepulciano, Uva di Troia.

\section{Conclusions}

Anthocyanins and flavonols are secondary metabolites useful for grape chemotaxonomy because of their dependence on genetic factors. The metabolomic approach was associated with the statistical analysis of qualitative and semi quantitative data. This approach proved to be useful tool for the varietal characterization of the vine. Specifically, it was possible to clearly divide the twenty Italian native varieties into five groups according to their polyphenolic profile. This result may be supplemented and confirmed by the analysis of different vintages.

\section{References}

[1] Boulton, R. Am. J. Enol. Vitic. 52 (2): 67-87 (2001)

[2] De Pascual-Teresa S., Sanchez-Ballesta M.T. Phytochem. Rev. 7 (2):281-299 (2008)

[3] Flamini R., De Rosso M., Bavaresco L. J. Anal. Methods Chem. ID 350259 (2015)

[4] Flamini R., De Rosso M., De Marchi F., DallaVedova A., Panighel A., Gardiman M., Maoz I., Bavaresco L. Metabolomics 9: 1243-1253 (2013)

[5] Hammer, Ø., Harper, D.A.T., Ryan, P.D. 2001 Paleontological statistics software package for education and data analysis. Palaeontologia Electronica 4(1): 9pp.

[6] Manach C., Scalbert A., Morand C., Rémésy C., Jiménez L. Am. J. Clin. Nutr. 79 (5): 727-747 (2004)

[7] Mattivi F., Guzzon R., Vrhovsek U., Stefanini M., Velasco, R. J. Agric. Food Chem. 54 (20):7692-7702 (2006) 